\title{
8.10.4.3 Tritium Permeation and Recovery for the Flibe/He Blanket Design
}

A study of tritiun peraetion and recovery for molten salt for the fusior: breeder is reported in Ref. 8.20-14. The results are directly relevant to the fusion electric case. This atudy asames tritium to be a gas dissolved in molten salt, with TF formation suppresser. Tritium permeates readily through the hot steel tubes of the reactor and steam generator and will leak into the oteam aystem at the rate of about one gram per day is the absence of special permeation barriers, ssumi. that 17 of the helium coolant flow rate is processed for tritium recovery i $90 \mathrm{Z}$ efficiency per pess. Tritiated water in the oteam oystem is a personnel hazard at concentration levels well below one part per willion a.d this level would soon be reached without costly isotopic processing. Alternatively, incluoting a combination of permeation barriers on reactor and steam generator tubes and molten alt processing is estimated to $r$ tuce the leak rate into the steam system by over two orders of magnitude. Fr the option with the lowest estimated leak rate, $55 \mathrm{Ci} / \mathrm{d}$, it may be poss... $1 \mathrm{E}$ to purge the stean system continously to prevent tritiated water buildup. At best, isotopic separation of dilute tritioted uter way not be necessary and for higher leak-rate options the isotopic p:ocessing rate can be reduced.

The proposed permeation barrier for the reactoz tubes is a $10 \mu \mathrm{m}$ layes of tungsten which, in principle, will reduce tritium blanket permeation by a factor of about 300 below the bare-steel rate. A research and development effort is needed to prove feasibility or to develop alternative barriers. The partial pressure of tritium gas dissolved in molten salt is high, easing the recovery process for which a flash-separator has been chosen. A $1 \mathrm{~mm}$ aluminum sleeve is proposed to suppress permeation through the steam generator tubes. This gives a calculated reduction factor of rore than 500 relative to bare steel, including a factor of 30 due to an assumed oxide layer.

To gain a becter understanding of perreation effects, equations describing steady-state tritium permeation without axial flow have been derived for a multi-layer tube wall within the blanket region. A layer of frozen salt is included, along with fluid boundary-layer resistances. Calculations of the partial-pressure distribution show significant differences for tubes irradiated at different power densities. Molten salt boundary-layer resistance can be important in the absence $n$ f a good 
permeation barrier, or for a lowpower tube coated with a nowinal $1 \mathrm{\mu m}$ tungsten barrier. Permeabilities of various netals are shown in Fig. 8.10-18. This nominal perweation barrier vill dominate the flow resistance, however, for medium or high power-densiby tubes closer to the firbt wall. Examination of the radial flux equarion shows complicated dependence on upstream partial pressure, which reduces to a linsar dependence at low pressures where Henzy's Law materials become flux limiters and equare-root dependence at high tritium partial pressures where Sievert' Law aterials are flux lititing.

An analytical codel has been developed to establish the tritium split between wall permestion and reactor-tube flow. Permeation barriers are shown in Fig. 8,10-19. The bartiers are shown on the outside of the tubes but could equally well be on the ingide. For the mo:z.al salt cubes the inside barrier would greatly reduce the critium inventory in the tube walls and further reduce the alread; very low corrosion rate. The tritium fraction escaping through the tube walls has been quantified for limiting cases of Henry's law and Sievert's Law barriers as fiux limiters. All parameters of design interest are explicitly included: tritium generation rates and solubility in salt, tube geometry, barrier permeation parameters, and molten salt processing rate ar.d recovery efïiciency.

The intermediate helium heat transfer loop has been treated as a wellmixed tank for analytica: purposes, with input frow the reactor, partial tritium recovery in a slipstream process loop, and sievert's Law permeation loss to the steam syster.

A combination of effective tritium permeation barriers are required on both blanket and steam generator tubes, together with substantial process rates for molten salt and helium systems, in order to hold tritium permeation into the steam system to $55 \mathrm{ci} / \mathrm{d}$. If this can be done, it way be feasible to simply purge the steam syster of incoming tritium with only minor environmental impact and personnel hazard from steam leaks, and without the necessicy of costly and hazardous isotopic processing to separate eritiaced and ordinary water.

A surprisingly thin $(10 \mu \mathrm{m})$ tungsten coating will, in principle, provide a good permeation barrier on the blanket rubes. The feasibility of, in fact, reducing eritium blanket perweation by a factor of 300 or so below the tare steel tube rate for some $10^{4} \mathrm{~m}^{2}$ of tube area will require a 
reaeszch and developaent effort. Other anterials or alloys way prove co be superior, probably at the price of greater thickness of coating.

A relatively thick i m aminum leeve vas selected to suppress permeation through the tean generator tubes. This gave a calculated reduction factor of more than 500 relative to bare eteel, including a factor of 30 due to an assumed oxide layer. This is essentially a brute force approach that may well be improved upon by the development of more sophisticated permeation bartiera.

Although we have focused attention on a tungsten bartier due to a remarkably low tritium permeability, beryllium and other low-permeability materials such es ceramics and cermets should be considered in $n$ barrier development probleil.

The tritium recovery oystem flow sheet is shown in Fig. 8.10-20. Due to the low solubility of tritium in the reducing salt, a simple flash separator will wllow removal of the tritium and other noncondensible cases, mainly helium. Tritium removal from helium is virtually a standard system. The bulk of the tritium is recovered as a hybride on a getter bed, with final cleanup accomplished by catalyzed oxidation and adsorption.

The diffusivity of tritium gas dissolved in molten salt will need to be measured, especially to verify whether or not the fluid boundary-layer barrier is realistic.

Finally, some definitive experimental vork on the kinetics of tritium gas conversion to tritiated water at low concentrations $i$ : helium is called for. Popular opinion has oscillated over the last decaje from an initial optimism chat thermodynamics would reduce the gas concentration to nil, to a current pessimicm that predicts no gas convergion at all in the main helium loop. The critical experiments remain to be done, both with "clean" walls and particulate-free helium, and in the presence of catalytic surfaces or other reaction promoters. The challenge is to demonstrate a method of drastically reducing tritium gas partial pressure in the internediate helium loop, and thus suppress permeation into the steam system. 


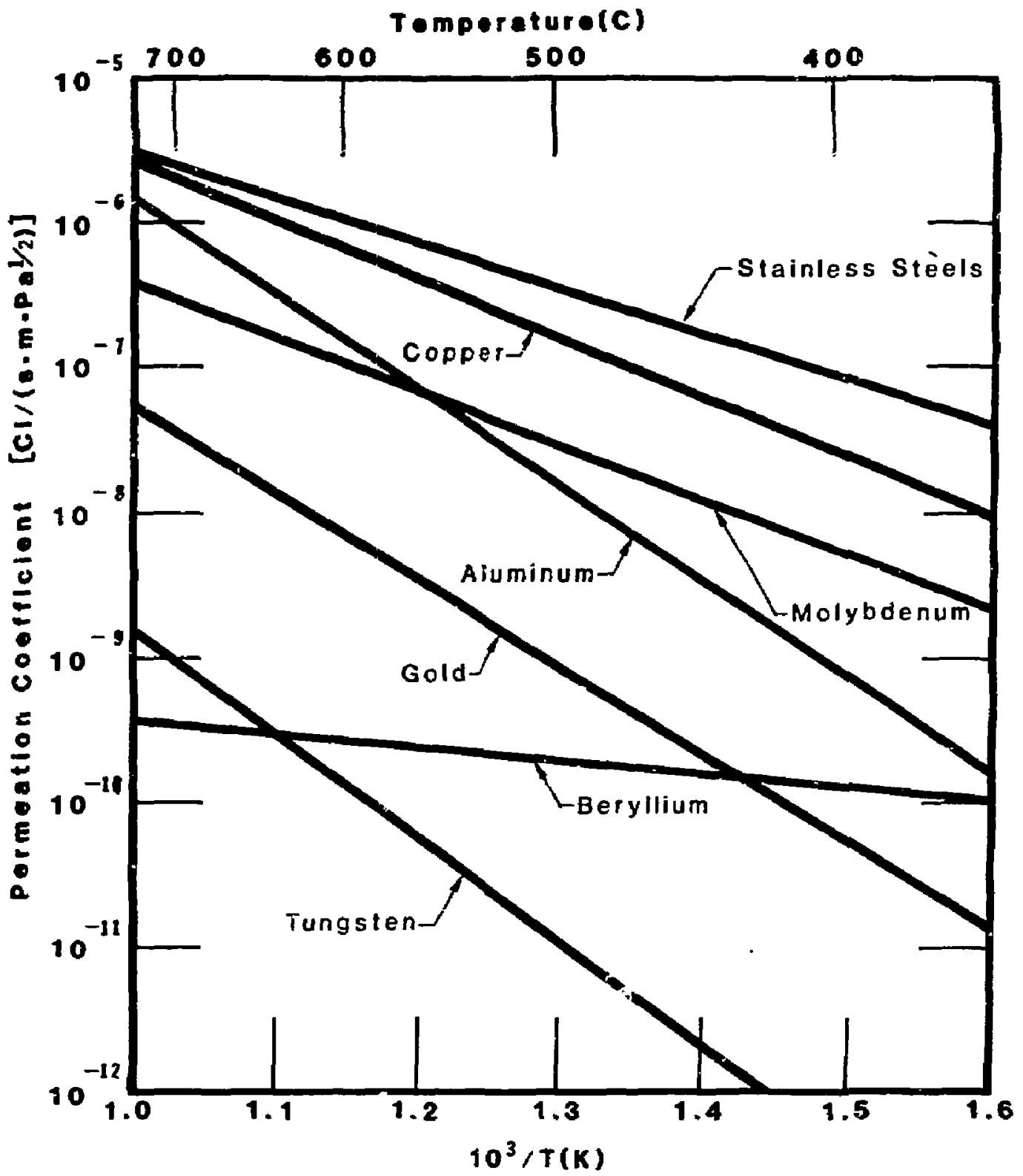

Figure $6.10-18$

Permeation coefficient of tritium through metals. 


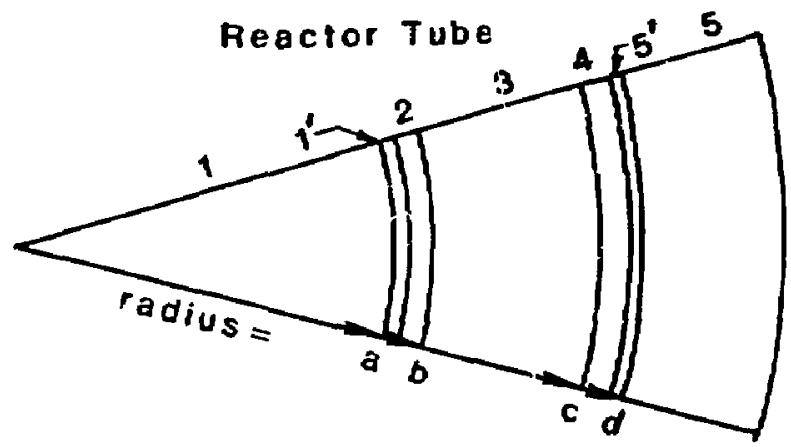

Steam Generator Tube

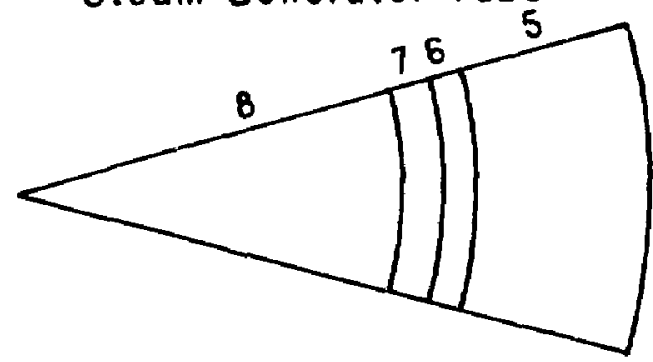

Figure 8.10-19

Perneation Geometry and Materials.

Reactor Tube:

1 colten balt

1 molten salt boundary layer

2 frozen sult

3 stainless cteel rube

4 perweation barrier (tungsten)

5' helium gas boundary layer

5 helium gas

\section{Steam Generacor Tube:}

5 helium gas

6 stainless steel tube

7 permeation barrier (aluminum)

B water/steam 


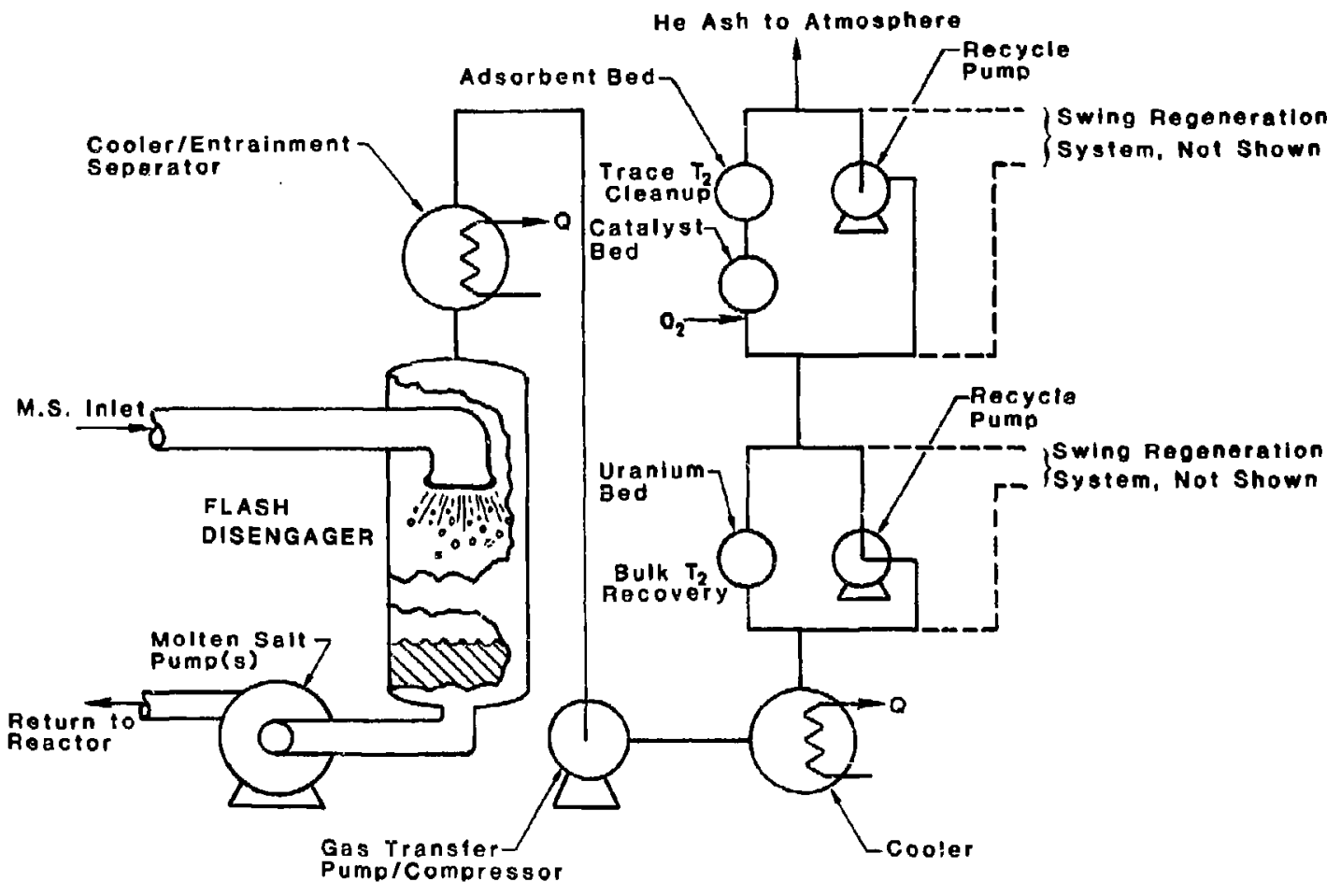

Figure 8.10-20. Molten salt tritium processing 